\title{
Importance of third moments of fluctuations of conserved charges in relativistic heavy-ion collisions ${ }^{\star}$
}

\author{
Masayuki Asakawa ${ }^{1, a}$, Shinji Ejiri ${ }^{2}$, and Masakiyo Kitazawa ${ }^{1}$ \\ 1 Department of Physics, Osaka University, 1-1 Machikaneyama, Toyonaka, Osaka 560-0043, Japan \\ 2 Department of Physics, Niigata University, Niigata 950-2181, Japan
}

\author{
Received: 20 October 2015 \\ Published online: 23 August 2016 \\ (C) The Author(s) 2016. This article is published with open access at Springerlink.com \\ Communicated by D. Blaschke
}

\begin{abstract}
We discuss the importance of third moments of conserved charges around their averages in relativistic heavy-ion collisions.
\end{abstract}

\section{Introduction}

Quantum chromodynamics (QCD) is believed to have a rich phase structure in the temperature $(T)$ and baryon chemical potential $\left(\mu_{\mathrm{B}}\right)$ plane. Lattice QCD calculations indicate that the chiral and deconfinement phase transitions are a smooth crossover on the temperature axis [1] while various models predict that the phase transition becomes of first order at high density [2]. The existence of the QCD critical point is thus expected.

One may expect that the singularity at the critical point, at which the transition is of second order, may cause enhancements of fluctuations if fireballs created by heavyion collisions pass near the critical point during the time evolution. Because of finite-size effects and critical slowing down, however, such singularities are blurred and its experimental conformation may not be possible $[3,4]$.

\section{Third moment of conserved charges around the averages}

Here we propose to employ signs of third moments of conserved charges around the averages, which we call, for simplicity, the third moments in the following, to infer the states created by heavy-ion collisions. In particular, we consider third moments of conserved quantities, the net baryon and electric charge numbers, and the energy,

$$
m_{3}(c c c) \equiv \frac{\left\langle\left(\delta N_{c}\right)^{3}\right\rangle}{V T^{2}}, \quad m_{3}(\mathrm{EEE}) \equiv \frac{\left\langle(\delta E)^{3}\right\rangle}{V T^{5}},
$$

\footnotetext{
* Contribution to the Topical Issue "Exploring strongly interacting matter at high densities - NICA White Paper" edited by David Blaschke et al.

a e-mail: yuki@phys.sci.osaka-u.ac.jp
}

where $N_{c}$ with $c=\mathrm{B}, \mathrm{Q}$ represent the net baryon and electric charge numbers in a subvolume $V$, respectively, $E$ denotes the total energy in $V, \delta N_{c}=N_{c}-\left\langle N_{c}\right\rangle$, and $\delta E=E-\langle E\rangle$. We also make use of the mixed moments defined as follows:

$$
m_{3}(c c \mathrm{E}) \equiv \frac{\left\langle\left(\delta N_{c}\right)^{2} \delta E\right\rangle}{V T^{3}}, \quad m_{3}(c \mathrm{EE}) \equiv \frac{\left\langle\delta N_{c}(\delta E)^{2}\right\rangle}{V T^{4}} .
$$

To understand the behaviors of these moments around the QCD phase boundary, we first notice that the moments eqs. (1) and (2) are related to third derivatives of the thermodynamic potential per unit volume, $\omega$, with respect to the corresponding chemical potentials and $T$. The simplest example is $m_{3}(\mathrm{BBB})$, which is given by

$$
m_{3}(\mathrm{BBB})=-\frac{\partial^{3} \omega}{\partial \mu_{\mathrm{B}}^{3}}=\frac{\partial \chi_{\mathrm{B}}}{\partial \mu_{\mathrm{B}}},
$$

where the baryon number susceptibility, $\chi_{\mathrm{B}}$, is defined as

$$
\chi_{\mathrm{B}}=-\frac{\partial^{2} \omega}{\partial \mu_{\mathrm{B}}^{2}}=\frac{\left\langle\left(\delta N_{\mathrm{B}}\right)^{2}\right\rangle}{V T} .
$$

The baryon number susceptibility $\chi_{\mathrm{B}}$ diverges at the critical point and has a peak structure around there.

Since $m_{3}(\mathrm{BBB})$ is given by the $\mu_{\mathrm{B}}$ derivative of $\chi_{\mathrm{B}}$ as in eq. (3), the existence of the peak in $\chi_{\mathrm{B}}$ means that $m_{3}(\mathrm{BBB})$ changes its sign there. Although the precise size and shape of the critical region are not known, various models predict that the peak structure of $\chi_{\mathrm{B}}$ well survives far along the crossover line. For also $m_{3}(c c \mathrm{E})$ and $m_{3}(c \mathrm{EE})$, a similar argument applies.

Once the negativeness of third moments is established experimentally, it is direct evidence of two facts: 1) the existence of a peak structure of corresponding susceptibility 


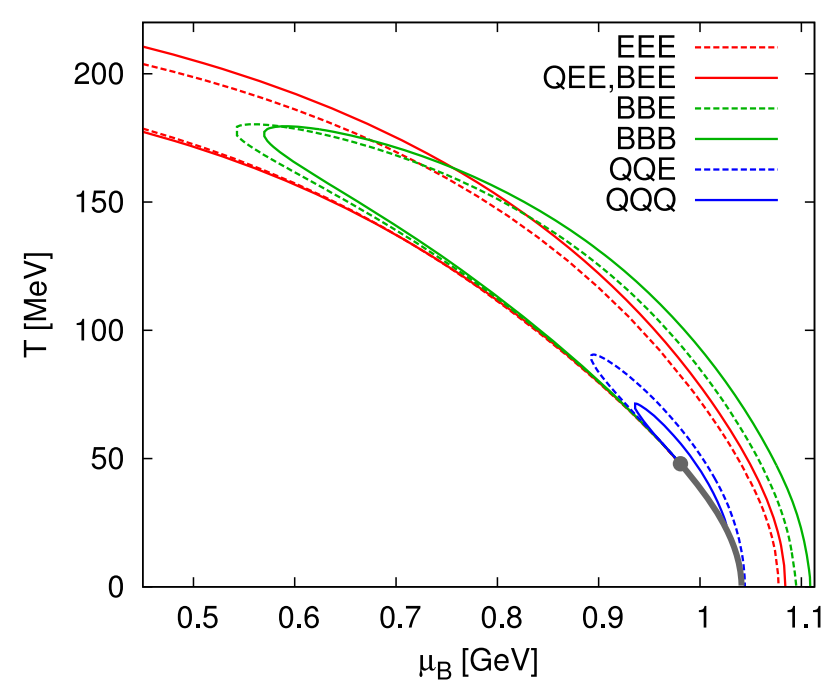

Fig. 1. Regions where third moments take negative values in the $T-\mu_{\mathrm{B}}$ plane. The regions are inside the boundaries given by the lines.

in the phase diagram of QCD, and 2) the realization of hot matter beyond the peak, i.e. the quark-gluon plasma, in heavy-ion collisions. We emphasize that this statement using the signs of third moments is free from any theoretical ambiguities and is a mathematical consequence [5].

The range of $\mu_{\mathrm{B}} / T$ where lattice simulations are successfully applied is limited to small $\mu_{\mathrm{B}} / T$ with the present algorithms. In particular, thermodynamics around the critical point cannot be analyzed with the Taylor expansion method. In order to evaluate the qualitative behavior of the third moments in such a region, one has to resort to effective models of QCD. To make such an estimate, here we employ the two-flavor Nambu-JonaLasinio model $[6,7]$ with the standard interaction $\mathcal{L}_{\text {int }}=$ $G\left\{(\bar{\psi} \psi)^{2}+\left(\bar{\psi} i \gamma_{5} \tau_{i} \psi\right)^{2}\right\}$, where $\psi$ denotes the quark field. For the model parameters, we take the values determined in ref. [6]; $G=5.5 \mathrm{GeV}^{-2}$, the current quark mass $m=$ $5.5 \mathrm{MeV}$, and the three-momentum cutoff $\Lambda=631 \mathrm{MeV}$. For the isospin symmetric matter, this model gives a firstorder phase transition at large $\mu_{\mathrm{B}}$.

The region where each moment becomes negative in the $T-\mu_{\mathrm{B}}$ plane is shown in fig. 1 . One sees that all the moments become negative on the far side of the critical point as it should be, whereas the extent of the region depends on the channel. The figure shows that areas with $m_{3}(\mathrm{BBB})<0$ and $m_{3}(\mathrm{BBE})<0$ extend to much lower $\mu_{\mathrm{B}}$ and much higher $T$ than the critical point. This suggests that even if the critical point is located at high $\mu_{\mathrm{B}}$ the negative third moments can be observed by heavy-ion collision expaeriments at relatively large collision energies.
It should be, however, remembered that the results in fig. 1, are obtained in an effective model. In particular, the model employed here gives the critical point at relatively low $T$ and high $\mu_{\mathrm{B}}$ [8]. If the critical point is at much lower $\mu_{\mathrm{B}}$, the areas with negative moments in fig. 1 should also move toward lower $\mu_{\mathrm{B}}$ and higher $T$.

\section{Conclusion}

In this short note, we emphasized the importance of the third moment of the conserved charges. The fluctuations of conserved charges do not change in the phase transition. However, note that they change during the hadron phase following the phase transition owing to diffusion [9]. So far, we have carried out all discussion in coordinate space. The values of fluctuations observed in momentum space are subject to thermal blurring owing to the thermal motion of charged particles [10]. Furthermore, it should be remembered that the simple correspondence between space time rapidity and rapidity as in Bjorken flow case does not exist any more at such low energy collisions as at NICA.

It cannot be emphasized too much that it is essential to cover as large $T-\mu_{\mathrm{B}}$ space by changing colliding energy to draw a figure like fig. 1 and get the full information on the QCD phase diagram. From this point of view, NICA project is as important as RHIC and LHC.

Open Access This is an open access article distributed under the terms of the Creative Commons Attribution License (http://creativecommons.org/licenses/by/4.0), which permits unrestricted use, distribution, and reproduction in any medium, provided the original work is properly cited.

\section{References}

1. T. Hatsuda, J. Phys. G 34, S287 (2007).

2. M.A. Stephanov, PoS LAT2006, 024 (2006).

3. B. Berdnikov, K. Rajagopal, Phys. Rev. D 61, 105017 (2000).

4. C. Nonaka, M. Asakawa, Phys. Rev. C 71, 044904 (2005).

5. M. Asakawa, S. Ejiri, M. Kitazawa, Phys. Rev. Lett. 103, 262301 (2009).

6. T. Hatsuda, T. Kunihiro, Phys. Rep. 247, 221 (1994).

7. T. Kunihiro, Phys. Lett. B 271, 395 (1991).

8. M. Asakawa, K. Yazaki, Nucl. Phys. A 504, 668 (1989).

9. M. Sakaida, M. Asakawa, M. Kitazawa, Phys. Rev. C 90, 064911 (2014).

10. Y. Onishi, M. Asakawa, M. Sakaida, M. Kitazawa, in preparation. 